\title{
Near-total Laryngectomy: An Oncologically Safe Alternative
}

\author{
${ }^{1}$ Arjun Dass, ${ }^{2}$ Surinder K Singhal, ${ }^{3}$ Nitin Gupta, ${ }^{4}$ Hitesh Verma
}

\begin{abstract}
Traditional treatment for advanced transglottic laryngeal and hypopharyngeal cancers is wide field laryngectomy. Patients who undergo wide field laryngectomy must also undergo voice rehabilitation with a prosthesis, electrolarynx or esophageal speech. These are associated with complications, such as bloating of the stomach, stenosis, aspiration and difficulty with hygiene of the TEP prosthesis. Near-total laryngectomy is a surgical treatment modality, where speech is maintained by way of a dynamic myomucosal shunt. It was specially designed to overcome the serious complications of adynamic tracheoesophageal shunt methods.
\end{abstract}

Keywords: Near-total laryngectomy, Myomucosal shunt, Interarytenoid area.

How to cite this article: Dass A, Singhal SK, Gupta N, Verma $\mathrm{H}$. Near-total Laryngectomy: An Oncologically Safe Alternative. Int J Phonosurg Laryngol 2014;4(1):30-32.

\section{Source of support: Nil}

Conflict of interest: None

\section{INTRODUCTION}

Near-total laryngectomy or Pearson procedure is a voicepreserving procedure which can be considered as an alternative to total laryngectomy for selected patients with lateralized, locally advanced cancers of the larynx and hypopharynx where speech is maintained by way of a dynamic myomucosal shunt created from the uninvolved tissues of the larynx. ${ }^{1}$ The dynamic shunt is a tunneled mucosal shunt between the trachea and pharynx. Its activity is regulated by preservation of the arytenoid cartilage, a variable portion of the vocal cord and recurrent laryngeal nerve on the side of the shunt creation. Such a tunnel will allow unilateral direction of air from lung to pharynx during phonation and will prevent aspiration since deglutition is a vagal mediated response which will induce contraction of tubed laryngeal musculature preventing aspiration. $^{2}$

${ }^{1}$ Professor and Head, ${ }^{2}$ Associate Professor, ${ }^{3,4}$ Assistant Professor

${ }^{1-4}$ Department of Otorhinolaryngology-Head and Neck Surgery Government Medical College and Hospital, Chandigarh, India

Corresponding Author: Hitesh Verma, Assistant Professor Department of Otorhinolaryngology—Head and Neck Surgery ENT Office, D Block, 3rd Floor, Government Medical College and Hospital, Chandigarh, India, Phone: 01722665253 , e-mail: hitesh_verma72@yahoo.com

\section{CASE REPORT}

A 60-year-old male patient presented with 2 months history of dysphagia, which was more for solids then liquids. He also had 1 month history of difficulty in breathing and hoarseness of voice. These symptoms were insidious in onset and gradually progressive. Indirect laryngoscopy showed an ulceroproliferative (UPG) growth involving left aryepiglottic fold (AEF) with pooling of secretions in left pyriform sinus (PFS) and fixity of left vocal cord. Neck examination revealed a $3 \times 3 \mathrm{~cm}$ firm, mobile lymph node at level III on left side. The remaining local examination and systemic evaluation for metastasis were normal. The FNA report was metastatic squamous cell carcinoma. Direct laryngoscopy under general anesthesia showed an UPG involving, left AEF and medial and lateral walls of left PFS. The apex of left PFS, interarytenoid area, left false vocal cord, left true vocal cord, postcricoid, cricopharynx and, right half of larynx and PFS were free of disease. The biopsy report was squamous cell carcinoma moderately differentiated type. After informed consent, the patient was planned for near-total laryngectomy with modified neck dissection (MND). Tumor was removed (Fig. 1) with adequate margins and with preservation of uninvolved larynx and pharynx (Fig. 2). Modified neck dissection Type III was done. Myomucosal shunt was created with interrupted sutures on $12 \mathrm{~F}$ Foley catheter (Fig. 3). The neopharynx was prepared with continuous intermittent locking extramucosal suture. The wound was closed in layers (Fig. 4). The patient was kept on intravenous antibiotics and nasogastric tube feeding for 10 days. The stomal sutures and stent were removed and oral trial was given on 10th postoperative day. There was transient aspiration. Voicing was done after 3 weeks which showed good results. The final histopathology examination showed resection free margins with positive neck nodes. The patient received radical chemoradiation. He is disease-free and under regular follow-up since 6 months.

\section{DISCUSSION}

The functions of larynx are protect the lower airway, respiration, production of sound, coughing, the Valsalva maneuver and control of ventilation, and acting as a sensory organ. Larynx is the second most common site for head and neck carcinomas after oral cavity. It is more common in 


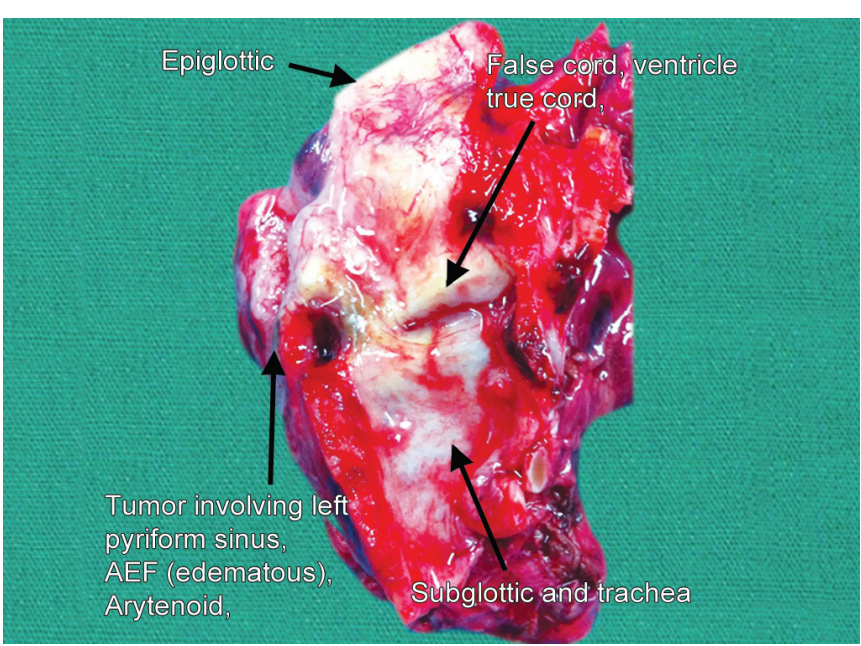

Fig. 1: Specimen

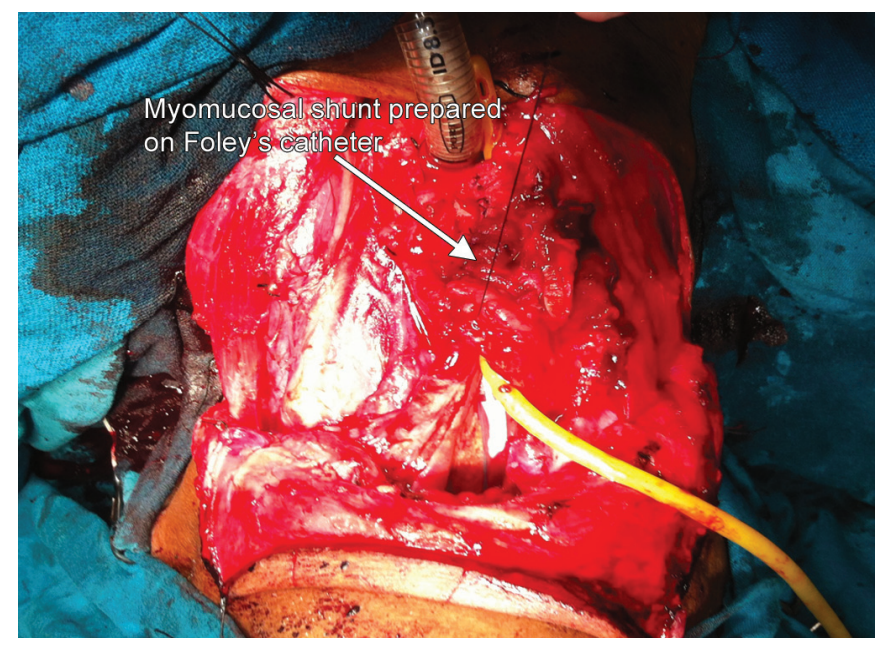

Fig. 3: Myomucosal shunt prepared on Foley's catheter with partial neopharynx

males and has a peak incidence during the sixth and seventh decades of life. The symptoms of laryngeal cancer include hoarseness, otalgia, dysphagia, dyspnea and weight loss. Alcohol and tobacco have synergistic carcinogenic properties, which directly contribute to most causes of laryngeal cancer. Most laryngeal cancers have a squamous cell histopathology (93\%).

The ultimate goal of every clinician treating laryngeal cancer is the extirpation of disease with the maintenance of voice and swallowing functionality. Traditional treatment for advanced transglottic laryngeal and hypopharyngeal cancers is wild field laryngectomy. The aphonic condition that the patient has to face after total laryngectomy has deep psychological and debilitating effects. In these cases, the options for speech are voice rehabilitation with a prosthesis, electro-larynx or esophageal speech. ${ }^{3-6}$ The problem with these rahabilitations are bloating of the stomach, dependency on cricopharyngeal muscle function, stenosis, aspiration and difficulty with hygiene of the TEP prosthesis. ${ }^{7}$ Whereas in

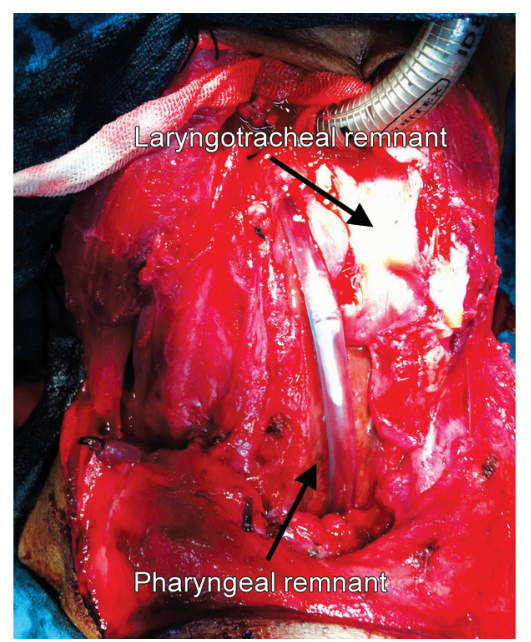

Fig. 2: Uninvolved larynx and trachea with pharynx

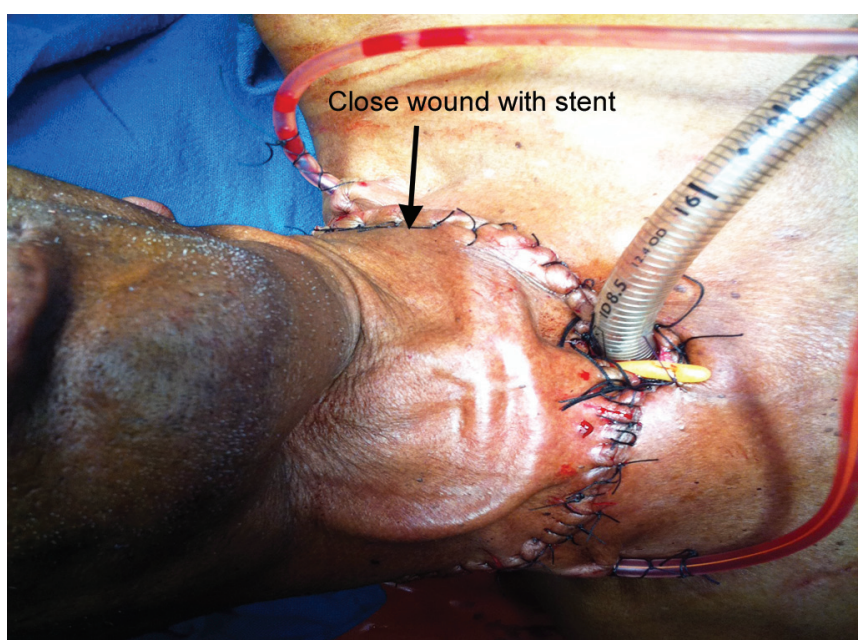

Fig. 4: Closed wound with Foley's catheter in situ

conservative laryngeal surgery, we can remove the necessary cancerous tissue without overly aggressive resection of the larynx and pharynx, thereby maintaining voice..$^{8-10}$ Conservative laryngeal surgeries have their own limitations. It becomes invalid in advanced stage disease, patients with poor pulmonary reserves, involvement of cricoid cartilages and elderly. Near-total laryngectomy (NTL) is a conservative procedure which can be a valid surgical option even with the above mentioned limitations. A patient who undergoes NTL speaks using an internal myomucosal shunt, which is lungpowered. The neoglottis is maintenance free; it, however, requires a tracheostomy for breathing.

Indications for NTLs include laryngeal and hypopharyngeal tumors that cause unilateral vocal cord paralysis where postcricoid and interarytenoid areas are free of disease and the contralateral arytenoid is salvageable in fresh or postradiation therapy.

Careful determination of tumor dimensions and its spread is the key factor for success in near total laryn- 
gectomy. ${ }^{11}$ Computed tomography scanning is very helpful preoperatively to evaluate submucosal extension, pre-epiglottic space involvement and cartilage invasion. Pulmonary function tests are required to determine pulmonary reserve. Panendoscopy should be performed to rule out synchronous primary cancers. Postoperative radiation is indicated in T3 or T4 stage, positive margin, multiple lymph node involvement, perineural invasion and evidence of vascular spread.

In NTL, the local control of cancer was similar to that expected with total laryngectomy. Hence, it is a valid alternative for extended laryngeal and neighboring cancers with an acceptable morbidity and success rate for voice preservation. ${ }^{12}$

\section{CONCLUSION}

Near total laryngectomy is a voice conservative surgery which is oncologically safe for advanced but lateralized laryngeal hypopharyngeal cancer with acceptable morbidity.

\section{REFERENCES}

1. Pearson BW. Subtotal laryngectomy. Laryngoscope 1981; 91(11):1904-1912.

2. D'Cruz AK, Sharma S, Pai PS. Current status of near-total laryngectomy: review. J Laryngol Otol 2012;126(6):556-562.
3. Asai R. Laryngoplasty after total laryngectomy. Arch Otolaryngol 1972;95(2):114-119.

4. Staffieri M, Procaccin A, Steine W, Staffier A. Surgical rehabilitation of speech after total laryngectomy: the staffieri techniques. Laryngol Rhinol Otol 1978;57(6):477-488.

5. Singer MI, Blom ED. An endoscopic technique for restoration of voice after laryngectomy. Ann Otol Rhinol Laryngl 1980; 89(6):529-533.

6. Panje WR. Prosthetic vocal rehabilitation following laryngectomy: the voice button. Ann Otol Rhinol Larynol 1981;90(2): 116-120.

7. Gavilan J, Herranz J, Prim J, Rabanal I. Speech results and complications of near total laryngectomy. Ann Otol Rhinol Laryngol 1996;105(9):729-733.

8. Redaelli de Zinis LO, Ferrari L, Tomenzoli D, Premoli G, Parrinello G, Nicolai P. Post laryngectomy pharyngocutaneous fistula: incidence, predisposing factors and therapy. Head Neck 1999;21(2):131-138.

9. Bernaldez R, Garcia-Pallares M, Morera E, Lassaletta L, Del Palacio A, Gavilan J. Oncologic and functional results of near total laryngectomy. Otolaryngol Head Neck Surg 2003; 128(5):700-705.

10. Pradhan SA, D'Cruz AK, Pai PS. Near-total laryngectomy. Asian J Surg 2002;25(1):27-34.

11. Strome SE, Robey TC, Devaney KO, Krause CJ, Hogikyan ND. Subglottic carcinoma: review of a series and characterization of its patterns of spread. Ear Nose Throa J 1999;78(8):622-628.

12. De Paula Vernetta C, De la Fuente Arjona L, García Callejo FJ, Ramírez Sabio JB, Marco Algarra J. Near-total laryngectomy. Experience, complications and acoustic analysis. Acta Otorrinolaringol sp. 2005;56(9):423-427. 